\title{
A PROCEDURE TO SET PHASE AND AMPLITUDE OF THE RF IN THE SNS LINAC'S SUPERCONDUCTING CAVITIES
}

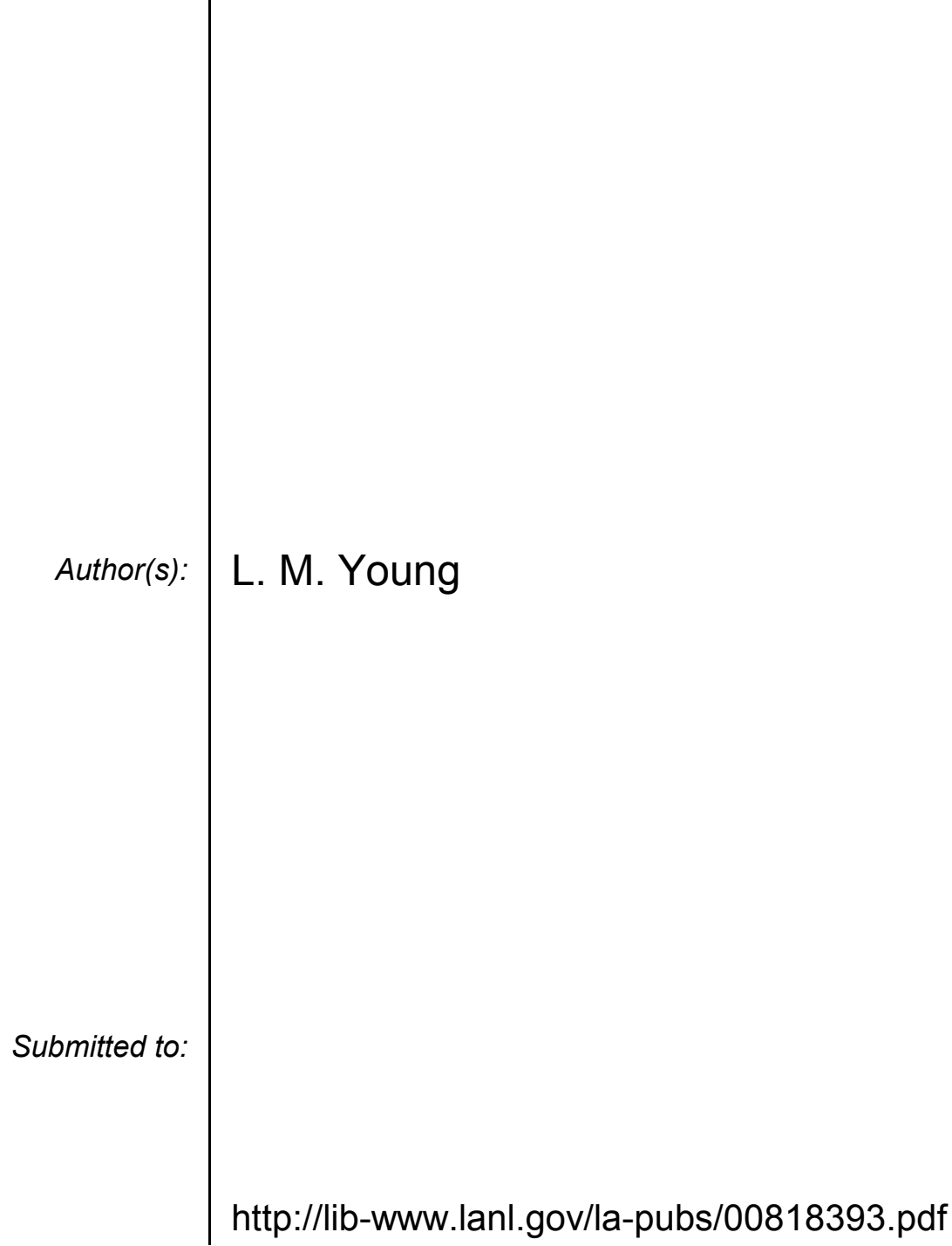

Los Alamos National Laboratory, an affirmative action/equal opportunity employer, is operated by the University of California for the U.S. Department of Energy under contract W-7405-ENG-36. By acceptance of this article, the publisher recognizes that the U.S. Government retains a nonexclusive, royaltyfree license to publish or reproduce the published form of this contribution, or to allow others to do so, for U.S. Government purposes. Los Alamos National Laboratory requests that the publisher identify this article as work performed under the auspices of the U.S. Department of Energy. Los Alamos National Laboratory strongly supports academic freedom and a researcher's right to publish; as an institution, however, the Laboratory does not endorse the viewpoint of a publication or guarantee its technical correctness. 


\title{
A PROCEDURE TO SET PHASE AND AMPLITUDE OF THE RF IN THE SNS LINAC'S SUPERCONDUCTING CAVITIES*
}

\author{
L. M. Young ${ }^{\dagger}$, Los Alamos National Laboratory, Los Alamos, New Mexico 87545
}

\begin{abstract}
This paper describes a procedure to set the phase and amplitude of the RF fields in the Spallation Neutron Source (SNS) linac's superconducting cavities. The linac uses superconducting cavities to accelerate the $\mathrm{H}^{-}$ion beam from the normal conducting linac at $185 \mathrm{MeV}$ to a final energy of $\sim 1 \mathrm{GeV}$. There are two types of cavities in the linac, 33 cavities with a geometric beta of 0.61 and 48 cavities with a geometric beta of 0.81 . The correct phase setting of any single superconducting cavity depends on the RF phase and amplitude of all the preceding superconducting cavities. For the beam to be properly accelerated it must arrive at each cavity with a relative phase $\left(\phi_{\mathrm{s}}\right)$, called the synchronous phase, of about -20 degrees. That is, it must arrive early with respect to the phase at which it would gain the maximum energy by 20 degrees. This timing provides the longitudinal focusing. Beam particles arriving slightly later gain more energy and move faster relative to the synchronous beam particle. The problem is to set the phase and amplitude of each cavity in the linac so that the synchronous particle arrives at each cavity with the correct phase. The amplitude of each superconducting cavity will be adjusted as high as possible constrained only by the available RF power and the breakdown field of the cavity.
\end{abstract}

\section{COMMISIONING THE SNS SUPERCONDUCTING $\mathrm{H}^{-}$PROTON LINAC}

The superconducting linac [1] uses 6 cell cavities operating in the $\pi$ mode to accelerate the $\mathrm{H}^{-}$beam. At 185 $\mathrm{MeV}$, the energy at the beginning of the superconducting linac, the $\beta(\mathrm{v} / \mathrm{c})$ of the beam is 0.55 . The first section of the linac uses 6-cell cavities with a geometric $\beta$ of 0.61 . Thus, the beam must arrive at the first cell early and leave the last cell late with respect to design synchronous phase. The $33(\beta=0.61)$ cavities accelerate the beam to 387 $\mathrm{MeV}$. At this energy the beam $\beta$ is 0.706 and the beam must arrive late at the first cell and leave the last cell early. At $247 \mathrm{MeV}$ the beam $\beta$ matches the cavity $\beta$ and the coupling of the beam to the accelerating field in the cavity is the strongest. To make the most efficient use of each superconducting cavity the field amplitude will be set as high a possible. This amplitude will not be known until the accelerator is ready for operation and the

*Work supported by the Office of Basic Energy Science, Office of Science of the US Department of Energy, and by Oak Ridge National Laboratory.

†lyoung@lanl.gov accelerating field is calibrated. Using standard RF measurement techniques the field level can be calibrated to about $\pm 5 \%$. This level of accuracy is not good enough to set the phase and amplitude of the RF fields in each of the superconducting cavities before the beam is turned on even if there was a method to determine the absolute phase of each cavity. Therefore, I propose to use the beam to calibrate the field amplitude and phase of each superconducting cavity.

\subsection{Procedure to Commission SC Linac}

- Calibrate RF pickup probes of first cavity.

1 Tune the first cavity to $805 \mathrm{MHz}$ by exciting cavity with klystron, switching off the RF drive, and watching the phase shift of the RF in the cavity with the RF reference. When the phase shift during $\sim 100 \mu$ seconds is less than $\sim 2$ degree the cavity is tuned to $805 \mathrm{MHz} \pm 56 \mathrm{~Hz}$.

2 Use beam to excite cavity. Compare excitation of cavity with calculations (RF off.) With the cavity tuned as in step (1) the phase of accelerating fields in the cavity will be excited with the phase that extracts the maximum energy from the beam. (Exactly $180^{\circ}$ from the phase of maximum acceleration.)

3 Use date from step 2 to calculate coefficients used by low level RF (LLRF) system to display the amplitude in $\mathrm{MV} / \mathrm{m}$. The cavity phase could be displayed relative to the beam phase.

4 Amplitude and phase of cavity are set. Phase of cavity excited with beam is $\pm 180^{\circ}+\phi_{\mathrm{s}}$ of required phase. (Add $\pm 180^{\circ}+\phi_{\mathrm{s}}$ ).

- Set amplitude and phase setpoints of first cavity and turn on RF.

- Calibrate RF pickup probes of next cavity.

- Set amplitude and phase setpoints of next cavity. Turn on RF.

- Repeat previous steps until all cavities are set.

- Periodically check beam energy with time-of-flight measurements.

\section{PREREQUISITES FOR COMMISIONING THE LINAC}

Before the first superconducting cavity can be commissioned with the above procedure, the beam from the room temperature linac must be transported all the way through all the superconducting cavities to the beam dump. This will require adjusting the quadrupole doublets 
and steering magnets between cyromodules to lower strength than the nominal operating values. The beam from the room-temperature linac at $185 \mathrm{MeV}$ would be focused too strongly in the later parts of the linac by quads adjusted to focus the beam with energies up to 1000 $\mathrm{MeV}$. The steering magnets will also need to be adjusted during the commissioning procedure when the energy is increased in the higher energy region of the linac. To first order, the quads and steering magnets could be programmed to increase in proportion to the beam energy as it is increased by the commissioning procedure each time a superconducting cavity is brought up to power. However, the superconducting cavities will not be placed exactly on the centerline of the linac. A misaligned superconducting cavity tends to steer the beam a small amount which must be corrected by adjusting the steering magnets downstream from the superconducting cavity being brought up to power. In order to streamline the RF commissioning of the superconducting cavities an automatic steering algorithm should be implemented in the computer control system. This algorithm would use beam position information from the beam position monitors (BPM's). The BPM's also provide phase information that can be used to calculate beam energy from time-of-flight between BPM's. This information could then be used by another algorithm in the computer control system to adjust the quadrupole focusing magnets.

\section{SIMULATING THE BEAM-CAVITY INTERACTION}

The code TRAN simulates the transient response of the RF fields in a cavity from the beam current, the RF power from klystrons, RF losses in the cavity, and RF losses from the presence of the RF drive coupler. The code PARMELA [2] is contained within TRAN and is used to calculate the beam-field interaction in the cavity. The superconducting cavity has 6 cells electrically coupled through the bore. There are 6 modes in this cavity with the $\pi$ mode used to accelerate the beam. In this mode the axial electric field alternates sign from one cavity to the next. However, all six modes are needed to accurately simulate the transient response of the fields. Let $\bar{a}(q, t)$ be a complex number that represents the amplitude and phase of the $q^{\text {th }}$ mode at time $t$. The phase is with respect to the to the RF reference at frequency freq. The angular frequency $\omega=2 \cdot \pi \cdot$ freq . Then, $e^{-\omega_{q} \cdot \Delta t / 2 Q_{q}}$ represents losses in the cavity where $\omega_{q}$ is the angular frequency and $Q_{q}$ is the quality factor of the $q^{\text {th }}$ mode, and $\Delta t$ is a small increment of time. In this simulation with $Q_{q}$ equal to about $1.0 \mathrm{x}^{10}$ the losses in the cavity are negligible. However, $Q_{0}$ appears in the definition of $\beta$, the coupling to the RF drive coupler. $Q_{\text {loaded }}$ is the $Q$ loaded by the RF drive coupler. $\beta=\left(Q_{0} / Q_{\text {loaded }}\right)-1$, where $\mathrm{Q}_{0}$ is the unloaded $\mathrm{Q}$ of the accelerating mode. Each mode rotates in the complex plane with respect to the drive frequency. This rotation is represented by: $e^{i\left(\omega_{q}-\omega\right) \Delta t}$. For a small time step $\Delta \mathrm{t}$ and small frequency difference, this term is $\sim 1$. Which means that the phase of the RF fields do not change very much. It is this fact that makes it possible to measure the phase of the beam accurately with short high-current beam pulses.

\subsection{RF power induced voltage}

The forward RF power vector, $\bar{p} f$ is a complex number that represents the amplitude and phase of the RF drive. The RF power vector $\bar{p} t$ (see Eq. 1) represents the amplitude and phase of the RF transmitted out of cavity through the coupling to the RF driveline. $\beta$ is the coupling factor for the mode $n$.

$$
\bar{p} t=\sum_{q=0}^{N} \bar{a}(q, t) \cdot \mathrm{X}\left(q, N_{d}\right) \cdot \sqrt{\beta} .
$$

Where $N_{d}$ is the number of the cell that is coupled to the $\mathrm{RF}$ drive, and $\mathrm{N}$ is the total number of cells. Variable $\mathrm{X}(q, m)$ is the normalized amplitude in cell $m$ for the $q^{\text {th }}$ mode.

The values of $\mathrm{X}(q, m)$ are calculated by the computer code LOOP [3,4], which is based on coupled electrical circuits composed of resistance, inductance and capacitance (RLC) loops. $\mathrm{X}(q, m)$ represents the amplitude and sign of the RF field in each cavity for every mode in the coupled-cavity system. The net result (induced voltage vector) of the forward RF power and the reflected power on the $\mathrm{q}^{\text {th }}$ mode is given by Eq. 2 .

$$
\bar{P}(q)=(2 \cdot \bar{p} f-\bar{p} t) \cdot \sqrt{\beta} \cdot \mathrm{X}(q, N d) / \mathrm{X}(n, N d)
$$

\subsection{Beam induced voltage}

The beam-induced voltage in cell $m$ is represented by $\bar{B}(m)$ (see Eq. 3). Program PARMELA computes $\bar{B}(m)$ by summing the effect of each particle passing through the electric fields $\vec{E}(z, m)$ of the cavity. $\vec{V}(z)$ is the velocity of the particle at position $z . \phi_{0}$ is the phase of the RF fields.

$$
\bar{B}(m)=\frac{\text { charge } e \cdot \sum_{\text {particles }_{\mathrm{z}=0}} \int_{\int_{0}=L} \vec{V}(z) \bullet \vec{E}(z, m) \cdot e^{i\left(\omega \cdot z / \vec{V}(z)+\phi_{0}\right)} d z}{\int_{z}^{L} E_{z}(z, m) d z}
$$

The code SUPERFISH [5] calculates the electric field distribution $\vec{E}(z, m)$.

\subsection{Cavity fields}

With the values of $\bar{a}(q, t)$ (see Eq. 4) determined, the rest of the calculations can be performed. The reflected 
power can be calculated. Periodically, PARMELA simulations are performed to calculate the performance of the accelerator and update $\bar{B}(m)$. The field in cell $m$ of the cavity is given by Eq. 5 .

$$
\begin{aligned}
\bar{a}(q, t+\Delta t) & =\bar{a}(q, t) \cdot e^{\left\{-\omega_{q} / 2 \cdot Q_{q}+i \cdot\left(\omega_{q}-\omega\right)\right\} \cdot \Delta t} \\
& +\left\{\bar{P}(q)+\sum_{m=1}^{N} \bar{B}(m) \cdot \mathrm{X}(q, m) / N\right\} \cdot \Delta t \\
\overline{\vec{E}}(z, m, t) & =\vec{E}(z, m) \cdot \sum_{q} \bar{a}(q, t) \cdot \mathrm{X}(q, m)
\end{aligned}
$$

PARMELA is an integral part of TRANS. It transports the beam multiple times through the part of the accelerator simulated by TRANS. The evolution of the beam pulse is thereby simulated. TRANS provides PARMELA with the phase and amplitude of the field in the accelerating cells. PARMELA returns the beam drive to TRANS. TRANS also simulates the digital RF feedback system controlling the RF power feeding the superconducting cavities.

\subsection{Example of using the code TRANS}

Figure 1 shows the output screen of the code TRANS simulating the beam excitation of a superconducting cavity. The labels on this figure point out some of the information that is displayed by the code. This figure is a combination of 3 runs of the code with 3 different cavity tunes $(+100,0$, and $-100 \mathrm{~Hz}$. The important information contained in this figure is the phase and amplitude response of the cavity to the beam. Note that the amplitude is not sensitive to tuning errors, it is only reduced from 2.97 to $2.96 \mathrm{MV} / \mathrm{m}$ (a $0.3 \%$ reduction) for a $100 \mathrm{~Hz}$ error. The $100 \mathrm{~Hz}$ tuning errors result in less than $2^{\circ}$ phase error. If the cavity is tuned to an accuracy of 50 $\mathrm{Hz}$ this source can be ignored. The cavity can be tuned by

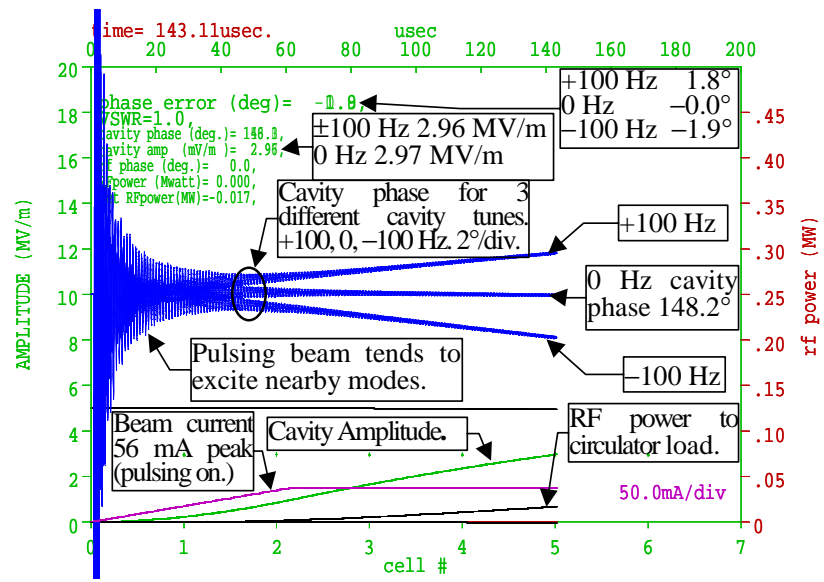

Figure 1; Example of phase response of superconducting cavity to $36 \mathrm{~mA}$ beam pulse when cavity is tuned $100 \mathrm{~Hz}$ high, correctly, and low $100 \mathrm{~Hz}$. The phase error at 143 $\mu$ second into pulse is $+1.8^{\circ}, 0^{\circ}$, and $-1.9^{\circ}$. Note that there is some ringing in the cavity that damps in about $60 \mu \mathrm{s}$, sufficient to see the effect of the cavity tune on the phase. turning on the RF power for a short time $(\sim 50 \mu \mathrm{s})$ then following the phase of the cavity for $\sim 100 \mu \mathrm{s}$. When the phase change is less than $\sim 2^{\circ}$ during this period the cavity is tuned to within $50 \mathrm{~Hz}$ of $805 \mathrm{MHz}$. When tuning the cavity with this method the cavity field remains low enough to ignore the Lorentz force detuning. The remaining source of tune error is caused by microphonics that is estimated to have $\sigma$ of $9 \mathrm{~Hz}$.

At least $60 \mu \mathrm{s}$ is required to get an accurate value of the phase because the contribution to the measurement from the other 5 modes in the superconducting cavity needs to damp away. Without beam chopping the excitation of the nearby modes is greatly reduced. The linac injects 1000 turns into the accumulator ring. The beam chopping is required by the accumulator ring to limit beam loss when the beam is extracted in one turn. The chopping frequency is $\sim 1000 \mathrm{kHz}$ and the nearest mode in the cavity is $\sim 800$ $\mathrm{kHz}$ lower in frequency then $\pi$-mode. In Fig. 1, increasing the chopper-on window ramps up the beam current to its maximum value in $60 \mu \mathrm{s}$. If the full beam current appears all at once, transients in the phase and amplitude of the room-temperature linac could affect the phase of the beam arriving at the superconducting accelerator. Thus, chopping should be used to keep the transients in the beam phase small.

\section{CONCLUSION}

With the method presented in this paper the phase of the beam can be measured with the SC cavities. Using TRANS the excitation of the cavity can be predicted given the measured current and beam energy. Thus the LLRF system can be calibrated to give the accelerating field of the SC cavities in MV/m. The phase and amplitude setpoints of each SC cavity can be set one at a time. However, with 90 cavities, the procedure would take quite a while if done manually one SC cavity at a time. This calls for an automatic procedure implemented in the control system to perform the setup of all the phase and amplitude setpoints for the SC linac.

\section{REFERENCES}

[1] J. Stovall, S. Nath, H. Takeda, J. Billen, L. Young, M. Lynch, D. Rees, J. Galambos, D. Jeon, D. Raparia, J. Wei, R. Sundelin, K. Crandall, C. Pagani, and P. Pierini, "Superconducting Linac for the SNS", Proc. International LINAC2000 (Monterey, August 21-25, 2000) pp. 605-607.

[2] L. M. Young, "PARMELA," Los Alamos National Laboratory report LA-UR-96-1835 (Revised February 27, 2001).

[3] http://laacg1.lanl.gov/laacg/componl.html

[4] S. O. Schriber, "Pi-Mode Structures -- Results and Implications for Operation", This conference.

[5] J. H. Billen, L. M. Young, "Poisson Superfish",LAUR-96-1834 (Revised February 21, 2001). 